\title{
Malnutrition in pre-dialysis chronic kidney disease patients in a teaching hospital in Southern Nigeria.
}

\author{
Adejumo Oluseyi ${ }^{1}$, Okaka Enajite ${ }^{2}$
}

1. Kidney Care Centre, Department of Internal Medicine

2. UBTH, Department of Internal Medicine

\begin{abstract}
Background: Malnutrition is a complication in chronic kidney disease (CKD) known to affect quality of life and prognosis although not often diagnosed. It is associated with rapid progression to end stage renal disease (ESRD) and mortality. Early identification and treatment will slow down progression to ESRD and mortality.

Objective: To determine the prevalence and pattern of malnutrition in pre-dialysis CKD patients in Southern Nigeria.

Methods: One hundred and twenty consecutive pre-dialysis CKD and 40 control subjects without CKD were studied. Data obtained from participants were demographics, body mass index (BMI), and aetiology of CKD. Indices used to assess presence of malnutrition were low BMI, hypocholesterolaemia and hypoalbuminaemia. Statistical significance was taken at 0.05 level.

Results: The mean age of the CKD subjects was $48.8 \pm 16$.6years with a male: female ratio of 1.7:1. Prevalence of malnutrition in the CKD subjects was $46.7 \%$, higher than $27.5 \%$ observed in the controls $(\mathrm{p}=0.033)$. Prevalence of malnutrition increased significantly across CKD stages 2 to $5(\mathrm{p}=0.020)$. It was significantly commoner in elderly patients $(\mathrm{p}=0.047)$ but not significantly different between males and females $(\mathrm{p}=0.188)$.

Conclusion: Malnutrition is common in pre-dialysis CKD patients even in early CKD stages. Prevalence of malnutrition increases with worsening kidney function and increasing age.

Keywords: Malnutrition, pre-dialysis, chronic kidney disease, Nigeria

DOI: http://dx.doi.org/10.4314/ahs.v16i1.31

Cite as: Oluseyi A, Enajite O. Malnutrition in pre-dialysis chronic kidney disease patients in a teaching Hospital in Southern Nigeria. Afri Health Sci. 2016;16(1): 234-241. http:/ / dx.doi.org/10.4314/abs.v16i1.31
\end{abstract}

\section{Introduction}

Chronic kidney disease has become a public health problem globally due to its increasing incidence, associated morbidity and mortality. ${ }^{1}$ Cardiovascular disease (CVD) is the leading cause of hospitalization and death in CKD patients at all stages. ${ }^{2}$ Hyperuricaemia , malnutrition-inflammation complex, hyperhomocystinaemia, calcium and phosphate abnormalities have been recognized as significant contributors to this cardiovascular burden. ${ }^{3}$ Despite the established awareness of their high cardiovascular risk, CKD patients still have inadequate risk factor assessment, modification and intervention. ${ }^{4}$

Malnutrition is a state of decreased body pool of protein with or without fat depletion caused at least partly by inadequate nutrient intake relative to nutrient demand
Corresponding author:
Adejumo Oluseyi,
kidney care centre,
internal medicine
Email: oludamilolaadejumo@gmail.com

and/ or improved by nutritional repletion ${ }^{5}$ There is a strong relationship between malnutrition, inflammation and atherosclerosis in $\mathrm{CKD}$, hence the term malnutrition-inflammation complex. It has been reported that the rapid atherosclerosis that occurs in advanced CKD is mediated by synergism of different mechanisms such as inflammation, malnutrition, oxidative stress and genetic components. ${ }^{6,7}$ This complex involving inflammation and hypoalbuminaemia are considered a part of emerging (non-traditional) cardiovascular risk factors in CKD that contributes to cardiovascular disease, mortality and rapid progression to end stage renal disease (ESRD). ${ }^{3,8}$

Malnutrition is one of the common features of chronic kidney disease (CKD), although not usually diagnosed. The prevalence of malnutrition in previous studies varied between $28-65 \%$ depending on criteria used in diagnosis. ${ }^{9-13}$ Majority of previous studies on malnutrition were carried out in end stage renal disease (ESRD) patients who were already on renal replacement therapy (RRT) with only a few in pre-dialysis ESRD patients. The causes of malnutrition in CKD are multi-factorial 
and include reduced food intake due to effect of uremia, reduced absorption of nutrients from oedematous gut, metabolic acidosis, increased protein loss during dialysis especially peritoneal dialysis, inflammation, oxidative stress, carbonyl stress and hormonal disorders. ${ }^{14-17}$

There are various nutritional indicators that could be used in assessing malnutrition in CKD either solely or in combination. Debates on the best mode of assessment of nutritional status in CKD patients is still on-going, but it is recommended that nutritional status be assessed with a combination of valid, complimentary measures rather than any single measure alone. ${ }^{18}$

Markers of nutritional status have been reported to be predictors of mortality in CKD patients. ${ }^{19-23}$ Early evaluation of pre-dialysis CKD patients for malnutrition, paying more attention to their diet, correcting metabolic disorders like acidosis may help mitigate development of cardiovascular disease, CKD progression and significantly reduce mortality before and after commencement of renal replacement therapy.

We set out to determine the prevalence and pattern of malnutrition in relation to age, gender, aetiology and severity of CKD among pre-dialysis CKD patients attending a teaching hospital in Southern Nigeria. This study adds to the body of knowledge on malnutrition in CKD because pre-dialysis patients at various stages of CKD were studied and not just ESRD. There is also a paucity of studies on malnutrition among CKD patients in Nigeria.

\section{Methodology}

The Renal Unit, Department of Internal Medicine of the hospital receives referral from within and outside the state of location. An average of 12 newly diagnosed CKD patients are seen in the Unit monthly while about half this number commence maintenance haemodialysis monthly.

This was a case control study spanning over a 2-year period (September 2011 to August 2013) that involved 120 consecutive pre-dialysis CKD patients and 40 age matched controls. Sample size was derived using the Fleiss formula for case-control study using the following information: ${ }^{24}$

Confidence interval $=95 \%$, power of study $=80 \%$, ratio of cases to control of $3: 1$, percentage of control exposed: $14.3 \%{ }^{25}$ and percentage of cases exposed:
$43.2 \%{ }^{26}$ This formula gave a minimum sample size of 93 for cases and 31 for control.

Ethical approval was obtained from the hospital ethics committee on research and informed consent was obtained from participants.

Inclusion criteria for CKD subjects was: adults aged $\geq 18$ years, patients with CKD stages $1-5$ yet to commence dialysis and those who consented to participate in the study. CKD patients already on dialysis therapy, those infected with HIV, hepatitis B virus or hepatitis $\mathrm{C}$ virus, those with chronic liver disease and those on statins was excluded from the study. Inclusion criteria for control subjects were adults aged $\geq 18$ years with normal renal function, absence of chronic liver disease, negative hepatitis B, hepatitis C and HIV status and not being on lipid lowering medications.

Demographic information including age and gender of participants was obtained. Patients and control subjects were interviewed, physically examined, investigated and aetiology of renal disease determined. Weight was measured using a weighing scale with subjects wearing light clothing. Height was measured using a stadiometer to the nearest centimeter with subjects not wearing shoes. The body mass index (BMI) was calculated using the formula; Weight / (Height) ${ }^{2}\left(\mathrm{~kg} / \mathrm{m}^{2}\right)$. About $10 \mathrm{mls}$ of venous blood after an 8 hour fast was obtained from patients to perform biochemical tests which included serum albumin, serum creatinine and fasting serum lipids. Glomerular filtration rate was estimated using CockcroftGault formula which has been previously validated in Nigerians. ${ }^{27}$

(140- Age in years) x weight $(\mathrm{kg})$ x 0.85 (for females)

72 x serum creatinine $(\mathrm{mg} / \mathrm{dl})$

\section{Definition of variables}

Estimated GFR was used to stage CKD as stage 1 (GFR of $\geq 90 \mathrm{mls} / \mathrm{min}$ with evidence of kidney damage), stage 2 (GFR of $60-89 \mathrm{mls} / \mathrm{min}$ with evidence of kidney damage), stage 3 (GFR of $59-30 \mathrm{mls} / \mathrm{min}$ with or without evidence of kidney damage), stage 4 (GFR of 15-29 mls/ min with or without evidence of kidney damage) and stage 5 (GFR $<15 \mathrm{mls} / \mathrm{min}$ with or without evidence of kidney damage). ${ }^{28}$ Malnutrition was defined in this study as any or a combination of these: serum albumin $<3.5 \mathrm{~g} / \mathrm{1}^{29}$, total cholesterol $<150 \mathrm{mg} /$ $\mathrm{dl}^{29}$ and $\mathrm{BMI}<18.5 \mathrm{~kg} / \mathrm{m}^{230}$ 


\section{Data analysis}

Data generated was entered and analysed using the statistical package for social sciences (SPSS) version 17.0. Results were presented in tabular form. Continuous variables were presented as means and standard deviation. Discrete variables were presented as percentages. Chi-square test was used to determine the significant associations between categorical variables while chisquare with trend was used where categorical variables were ordinal. Student t-test was used to compare mean values of the sub-groups. $\mathrm{P}$ values $<0.05$ were considered significant.

\section{Results}

A hundred and twenty CKD patients were recruited during the period of study, consisting of 76 males and 44 females. Forty aged matched healthy adults consisting of 25 males and 15 females were also recruited as control subjects. Most of the CKD subjects were below 65 years of age with only 23(19.2\%) aged 65 years and above. Hypertensive nephropathy was the commonest aetiology of CKD and accounted for $36.7 \%$ of patients. Majority of the CKD patients were in stages 3 and 4; accounting for $78.4 \%$ of CKD patients. (See table 1)

Table 1: Characteristics of Study Population

The mean age of CKD patients and controls were $48.8 \pm 16.6$ years and $45.0 \pm 14.9$ years respectively. CKD patients had significantly higher mean systolic BP, diastolic BP and serum creatinine compared to control subjects while estimated GFR and serum albumin was significantly lower in the CKD group compared to the control group. However, mean age and BMI were not significantly different between the CKD and control groups. A higher proportion of CKD patients had hypoalbuminaemia and low BMI compared to control subjects but the prevalence of hypocholesterolaemia was similar in both groups. The prevalence of malnutrition in the CKD subjects was $46.7 \%$ which was significantly higher than $27.5 \%$ observed in the control group $(\mathrm{p}=0.033)$. (See table 2$)$. 


\begin{tabular}{llll}
\hline Parameters & CKD $(\mathrm{N}=120)$ & Controls $(\mathrm{N}=40)$ & P value \\
& Mean $\pm \mathrm{Sd} / \mathrm{n}(\%)$ & Mean $\pm \mathrm{Sd} / \mathrm{n}(\%)$ & \\
Age(years) & $48.77 \pm 16.60$ & $45.00 \pm 14.90$ & 0.205 \\
Systolic BP(mmHg) & $163.45 \pm 28.57$ & $125.95 \pm 17.27$ & $<0.001$ \\
Diastolic BP(mmHg) & $97.09 \pm 19.70$ & $80.28 \pm 10.91$ & $<0.001$ \\
BMI(kg/m2) & $24.75 \pm 4.86$ & $26.10 \pm 3.82$ & 0.113 \\
Serum total cholesterol(mg/dl) & $178.66 \pm 42.06$ & $177.19 \pm 44.19$ & 0.911 \\
Serum albumin(g/dl) & $37.97 \pm 7.55$ & $46.37 \pm 8.03$ & $<0.001$ \\
Serum creatinine (mg/dl) & $3.06 \pm 1.58$ & $0.92 \pm 0.23$ & $<0.001$ \\
Estimated GFR(mls/min) & $31.03 \pm 16.04$ & $105.89 \pm 42.71$ & $<0.001$ \\
Hypoalbuminaemia & $27(22.5)$ & $0(0)$ & 0.002 \\
Hypocholesterolaemia & $32(26.7)$ & $11(27.5)$ & 0.920 \\
Low BMI & $6(5)$ & $0(0)$ & 0.337 \\
Malnourished & $56(46.7)$ & $11(27.5)$ & 0.033 \\
\hline BP (blood & & & \\
\hline
\end{tabular}

BP (blood pressure), BMI (body mass index), GFR (glomerular filtration rate). Low BMI defined as $B M I<18.5 \mathrm{~kg} / \mathrm{m}^{2}$. Malnourished defined as any or a combination of these: hypoalbuminaemia, low BMI and hypocholesterolaemia

The prevalence of malnutrition was significantly higher in patients $\geq 65$ years of age compared to those aged less than 65 years $(p=0.047)$. There was a significant increase in prevalence of malnutrition across CKD stages 2 to 5 from $30.8 \%$ to $69.2 \%$ ( $\mathrm{p}=0.020)$. There was no significant observed difference in the proportions of patients with malnutrition based on gender. Prevalence of malnutrition was highest in patients with hypertensive nephropathy compared to those with other aetiologies but this difference did not reach statistical significance (See table 3). 


\section{Table 3: Pattern of Malnutrition according to age group, gender, stage and aetiology of CKD}

\begin{tabular}{|c|c|c|c|}
\hline & $\begin{array}{l}\text { Malnourished } \\
\mathrm{n}(\%)\end{array}$ & $\begin{array}{l}\text { Not malnourished } \\
\mathrm{n}(\%)\end{array}$ & $P$ value \\
\hline \multicolumn{4}{|l|}{ Age group } \\
\hline$<65$ years & $41(42.3)$ & $56(57.7)$ & 0.047 \\
\hline$\geq 65$ years & $15(65.2)$ & $8(34.8)$ & \\
\hline \multicolumn{4}{|l|}{ Gender } \\
\hline Male & $24(54.5)$ & $20(45.5)$ & 0.188 \\
\hline Female & $32(42.1)$ & $44(57.9)$ & \\
\hline \multicolumn{4}{|l|}{ CKD stage } \\
\hline 2 & $4(30.8)$ & $9(69.2)$ & \\
\hline 3 & $19(38)$ & $31(62)$ & 0.020 \\
\hline 4 & $24(54.5)$ & $20(45.5)$ & \\
\hline 5 & $9(69.2)$ & $4(30.8)$ & \\
\hline \multicolumn{4}{|l|}{ Aetiology } \\
\hline Diabetic nephropathy & $15(41.7)$ & $21(58.3)$ & \\
\hline Hypertension & $23(52.3)$ & $21(47.7)$ & 0.807 \\
\hline CGN & $15(45.5)$ & $18(54.5)$ & \\
\hline Others & $3(42.9)$ & $4(57.1)$ & \\
\hline
\end{tabular}

\section{Discussion}

The prevalence of malnutrition in pre-dialysis CKD patients in this study was $46.7 \%$ which was significantly higher than $25.7 \%$ observed in controls with normal renal function. The prevalence of malnutrition in previous reports varied widely between $28-65 \% .^{9-13}$ This is due to differences in these studies in terms of criteria used in diagnosis and study subjects whether they were on maintenance dialysis or not.

Prakash et al studied pre-dialysis patients in India and found a high prevalence of $65 \% .{ }^{13}$ This high prevalence compared to findings in the index study could be explained thus. Firstly, only patients in stage $5 \mathrm{CKD}$ who are expected to have more severe disease, were studied while we looked at patients with all stages of CKD. Secondly, more tools for nutritional assessment were used including subjective global assessment (SGA) and skin triceps thickness compared to tools used in our study. Kadiri et al in Morocco found 29\% prevalence of malnutrition among patients on haemodialysis. ${ }^{10}$ This low prevalence compared to findings in our study could be because only BMI was used to assess the patients for malnutrition. 
This study also showed that malnutrition starts early in CKD and progressively increases across CKD stages 2 to 5 . This was similar to other reports both in paediatric and adult CKD subjects. ${ }^{31,32}$ As renal function declines, anorexia, loss of appetite, reduced food intake, vomiting, diarrhea become more prominent symptoms in these patients, hence predisposing them to malnutrition. This also tends to be worsened by the dietary restriction placed on these patients coupled with inadequate nutritional status monitoring. ${ }^{32}$ The effect of hormonal imbalance has been reported to contribute significantly to malnutrition in the latter stages of $\mathrm{CKD} .^{33}$

Malnutrition was significantly higher in elderly CKD patients compared to younger and middle aged patients in this study. This finding was similar to previous reports. ${ }^{11,12}$ This pattern is not surprising because aging is associated with malnutrition in the elderly even without CKD ${ }^{34}$ Decline in growth hormone and insulin growth factor-1, accumulation of free radicals, reduced immunity and chronic inflammation that occur with aging are factors contributing to malnutrition in elderly CKD patients. $^{35,36}$

Malnutrition was commoner in males compared to females in CKD subjects in this study though this was not statistically significant, similar to the report by Tayyem et al. ${ }^{37}$ There has been observation that loss of muscle mass and protein depletion is higher in male CKD patients, though the reason is not well understood. ${ }^{38} \mathrm{Mal}-$ nutrition was highest in patients with hypertensive nephropathy compared to other aetiologies, even though diabetes mellitus is an established risk factor for malnutrition in CKD.

The consequences of malnutrition include reduced quality of life, sleep disorder, depression, erythropoietin hyporesponsiveness, increased susceptibility to infections and aggravation of heart failure..$^{39-42}$ There is therefore, justification to incorporate nutritional assessment as part of managing CKD patients even in early stages as this study showed that malnutrition starts early in the course of CKD.

Whether nutritional interventions improve nutritional status and reduce cardiovascular complications is still a subject of debate. Supplementation of low protein diet in pre-dialysis CKD patients also reduced nitrogeneous product retention, acid-base imbalance, delayed initiation of RRT and preserved nutritional status in these patients. $^{43}$

\section{Limitation of study}

Subjective global assessment was not used in this study as part of the diagnostic stool for malnutrition, however it was reported that serum albumin correlated well with SGA scores in the diagnosis of malnutrition in CKD patients. ${ }^{44}$ This was a cross-sectional study, hence the long term effect of malnutrition on mortality in these patients could not be ascertained. The ratio of cases to control used in this study was 3:1 due to limitation of funds because this study was self- sponsored.

\section{Conclusion}

Malnutrition is common in pre-dialysis CKD patients even in early stages. The prevalence of malnutrition increases with increasing age and worsening kidney function. Nutritional status should be assessed early and regularly in these patients.

\section{Conflict of interest disclosure}

There is no conflict of interest.

\section{References}

1. Rayner HC, Pisoni RL, Bommer J, Canaud B, Hecking $\mathrm{E}$, Locatelli $\mathrm{F}$ et al. Mortality and Hospitalization in haemodialysis in five European countries. Results from dialysis outcome and practice patterns study (DOPPS). Nephrol Dial Transplant 2004; 19:108-120.

2. Alebiosu CO, Ayodele OE. The global burden of chronic kidney disease and the way forward. Ethn Dis. Summer 2005;15:418-423.

3. Locatelli F, Pozonni P, Tentori F, Del Vecchio L Epidemiology of cardiovascular risk factors in patients chronic kidney disease. Nephrol Dial Transplant 2003;18 (suppl7):2-9.

4. Agesh N, Navekar SA, Feffer AP. Cardiovascular risk in chronic kidney disease. Kid Int 2004;66:11-15.

5. Kovesdy C, Kopple JD, Kalandar-Zadeh K. Management of protein energy wasting in non-dialysis-dependent chronic kidney disease: reconciling low protein intake with nutritional therapy. Am J Clin Nutr 2013;97 (6):1163-1177.

6. Kalantar-Zadeh K, Ikizler TA, Block G, Avram MM, Kopple JD. Malnutrition inflammation complex syndrome in dialysis patients: causes and consequences. Am J Kidney Dis 2003;42:864-881.

7. Stenvinkel P, HeimburgerO, Paultre F, Diczfalusy U, Wang T, Berglund $\mathrm{L}$ et al.Strong association between malnutrition, inflammation, and atherosclerosis in chronic renal failure. Kidney Int 1999;55:1899-1911.

8. Taal MW, Brenner BM. Predicting initiation and pro- 
gression of chronic kidney disease: Developing renal risk scores. Kidney Int 2006:70;1694-1705.

9. Lawson JA, Lazarus R, Kelly JJ. Prevalence and prognostic significance of malnutrition in chronic renal insufficiency. Ren Nutr. 2001;11(1):16-22.

10. Kadiri ME, Nechba RB, Oualim Z. Factors predicting malnutrition in hemodialysis patients. Saudi J Kidney Dis Transpl 2011;22:695-704

11. Cianciaruso B, Brunori G, Kopple JD, Traverso G, Panarello G, Enia $G$ et al. Crosssectional comparison of malnutrition in continuous ambulatory peritoneal dialysis and hemodialysis patients. Am J Kidney Dis. 1995;26(3):475-486.

12. Tayyem RF, Mrayyan MT. Assessing the prevalence of malnutrition in chronic kidney disease patients in Jordan. J Ren Nutr . 2008;18(2):202-209.

13. Prakash J, Raja R, Mishra RN, Vohra R, Sharma N, Wani IA et al.High prevalence of malnutrition and inflammation in undialyzed patients with chronic renal failure in developing countries: A single centre experience from eastern India. Renal Failure 2007;29(7):811816

14. Kalantar-Zadeh K, Ikizler TA, Block G, Avram MM, Kopple JD. Malnutrition inflammation complex syndrome in dialysis patients: causes and consequences. Am J Kidney Dis 2003;42:864-881

15. Ballmer PE, McNurlan MA, Hulter HN, Anderson SE, Garlick PJ, Krapf R. Chronic metabolic acidosis decreases albumin synthesis and induces negative nitrogen balance in humans. J Clin Invest 95: 39-45, 1995

16. Lecker SH, Goldberg AL, Mitch WE. Protein degradation by the ubiquitinproteasome pathway in normal and disease states. J Am Soc Nephrol. 2006;17:1807-1819 17. Cueto Manzano AM. Hypoalbuminemia in dialysis. Is it a marker for malnutrition or inflammation? Rev Invest Clin. 200;53(2):152-158.

18. K/DOQI, National Kidney Foundation. Clinical practice guidelines for nutrition in chronic renal failure. Am J Kidney Dis 2000;35:1-140.

19. Leinig CE, Moraes T, Ribeiro S, Riella MC, Olandoski M, Martins $\mathrm{C}$ et al. Predictive value of malnutrition markers for mortality in peritoneal dialysis patients. J Ren Nutr. 2011;21(2):176-183

20. I-Wen W, Kuang-Hung H, Chin-Chan L, Chiao-Yin $\mathrm{S}$, Heng-Jung $\mathrm{H}$, Ming -Jui $\mathrm{H}$ et al. Re-evaluating the predictive roles of metabolic complications and clinical outcome according to estimated GFR levels- a four years prospective cohort study in Taiwan. BMC Nephrology 2013;14:92:doi:10.1186/1471-2369-14-92

21. Cooper BA, Penne EL, Bartlett LH, Pollock CA.
Protein malnutrition and hypoalbuminemia as predictors of vascular events and mortality in ESRD. $A m \mathrm{~J}$ Kidney Dis. 2004;43(1):61-66.

22. Leavey SF, Strawderman RL, Jones CA, Port FK, Held PJ. Simple nutritional indicators as independent predictors of mortality in hemodialysis patients. $A m \mathrm{~J}$ Kidney Dis. 1998;31(6):997-1006.

23. Kovesdy CP, Anderson JE, Kalantar-Zadeh K. Paradoxical association between body mass index and mortality in men with CKD not yet on dialysis. $A m \mathrm{~J}$ Kidney Dis. 2007 ;49(5):581-91

24. Fleiss JL. Statistical methods for rates and proportions. 2nd ed. New York, NY: John Wiley \& Sons; 1981 25. Rotimi C, Okosun I, Johnson L, Owoaje E, Lawoyin $\mathrm{T}$, Asuzu $\mathrm{M}$ et al. The distribution and mortality impact of chronic energy deficiency among adult Nigerain men and women. European Journal of clinical nutrition 1999;53:734-739

26. Agaba EI, Agaba PA. Prevalence of malnutrition in Nigeria with chronic renal failure. IJUN 2004:36(1):8993

27. Abefe SA, Abiola AF, Olubunmi AA, Adewale A. Utility of predicted creatinine clearance using MDRD formula compared with other predictive formulae in Nigerian patients. Saudi J Kidney Dis Transpl. 2009; 20:86-90.

28. Kidney Diseaes Improving Global Outcome (KDIGO) 2012 Clinical Practice Guideline of evaluation and management of CKD. Kidney Int Supplements 2013; 3:1-50.

29. Kulmann MK, Kribben A, Wittwer M, Horl WA. OPTA- malnutrition in chronic renal failure. Nephrol. Dial. Transplant 2007; 22(3):13-19

30. Clinical guidelines on the identification, evaluation and treatment of overweight and obesity in adults - The evidence report. National Institutes of Health. Obes Res 1998;6(2):51-209

31. Olowu WA, Adefehintin O, Aladekomo TA. Epidemiology and clinicopathologic outcome of paediatric chronic kidney disease in Nigeria; a single centre experience. AJNT 2013;6(2):105-113

32. Kopple JD, Greene T, Chumlea WC, Hollinger D, Maroni BJ, Merrill D et al. Relationship between nutritional status and the glomerular filtration rate: result from the MDRD study. Kidney Int. 2000;57(4):1688-1703 33. Yashpal PJ, Vijah K. Protein energy wasting in chronic kidney disease: An update with focus on nutritional interventions to improve outcomes. Indian J Endocrinol Metab. 2012; 16(2): 246-251.

34. Hickson M. Malnutrition and ageing. Postgrad Med J 2006;82:2-8 
35. Fedarko NS. The biology of aging and fraility. Clin Geriatr Med 2011;27:27-37

36. Perrini S, Laviola L, Carreira MC, Cignarelli A, Natalicchio A, Giorgino F. The GH/IGF-1 axis and signaling pathways in the muscle and bone: Mechanisms underlying age-related skeletal wasting and osteoporosis. J Endocrinol 2010;205:201-210

37. Tayyem RF, Mrayyan MT. Malnutrition and anthropometric and biochemical abnormalities in end stage renal disease patients. Saudi Med J 2007;28(10):1575- 1581 38. Marcen R, Teruel JL, de la Cal MA, Gamez C. The impact of malnutrition in morbidity and mortality in stable haemodialysis patients. Spanish Cooperative Study of Nutrition in Haemodialysis. Nephrol Dial Transplant 1997;12:2324-2331

39. Iyasere O, Brown EA Determinants of quality of life in advanced kidney disease: time to screen? Postgrad Med J. 2014;90(1064):340-7.
40. Akgul A, Bilgic A, Sezer S et al. Effect of protein energy malnutrition on erythropoietin requirement in maintenance haemodialysis patients. Hemodial Int 2007 11:198-203

41. Pecoits-Filho R, Lindholm B, Stenvinkel P. The malnutrition, inflammation and atherosclerosis (MIA) syndrome-the heart of the matter. Nephrol Dial Transplant 2002:17(11);28-31

42. Pecoits-Filho R, Lindholm B, Stenvinkel P. The malnutrition, inflammation and atherosclerosis (MIA) syndrome-the heart of the matter. Nephrol Dial Transplant 2002:17(11);28-31

43. Mircescu G, Garneata L, Stancu SH, Capusa C. Effect of supplemented hypoproteic diet in chronic kidney disease. J Ren Nutr. 2007;17(3):179-188.

44. Enia G, Sicuso C, Alati C, Zoccali C, Pustorino D, Biondo A. Subjective global assessment of nutrition in dialysis patients. Nephrol. Dial. Transplant 1993; 8(10):1094-198 